\title{
SMART CROSSWALKS: A SAFE ALERT SYSTEM THAT MINIMIZES THE CONFRONTATION BETWEEN VEHICLES AND PEDESTRIANS
}

\author{
João Branquinho ${ }^{1}$, Carlos Senna ${ }^{2}$ and André Zúquete ${ }^{3}$ \\ ${ }^{1} I T$, University of Aveiro, Portugal \\ ${ }^{2}$ Instituto de Telecomunicações, Portugal \\ ${ }^{3}$ DETI / IEETA / IT, University of Aveiro, Portugal
}

\begin{abstract}
A key characteristic of Smart Cities is the ability to reduce conflicts between different agents coexisting in a dynamic system, such as vehicles and pedestrians. This paper describes a system to augment the awareness of vehicle drivers regarding the presence of pedestrians in nearby crosswalks. To address this need, the system interconnects crosswalks to Road Side Units (RSUs), and RSUs to vehicles, in order to spread to vehicles the information about pedestrians in crosswalks. To prevent false information spreading, RSUs authenticate the alert messages they broadcast and all vehicles can validate them. This poses strong security requirements, such as non-repudiation of alert messages, as well as strong real-time requirements, such as minimum message propagation delays among vehicles approaching a crosswalk of interest. Because of this, the system minimizes the usage of asymmetric ciphers, which are fundamental to assure non-repudiation but increase performance penalties, and uses mostly symmetric ciphers for source authentication. To do so, we designed a new protocol, called Nimble Asymmetric Cryptography (NAC), which is useful to broadcast authenticated, implicit messages.
\end{abstract}

\section{KEYWORDS}

Smart Cities, Security, Smart Crosswalks

\section{INTRODUCTION}

Smart cities are a new reality, and an important branch of smart cities are Vehicular Ad-Hoc Networks (VANETs), where interconnected vehicles interact among themselves, with citizens and with the city itself. Currently, in Porto city, Portugal, buses interact among themselves and with the city itself in order to provide several services, such as Internet connectivity or information about the estimated time of arrival (Santos et al., 2018).

The goal of our work is to help car drivers to get alerts relatively to the presence of pedestrians in a crosswalk they are heading to. For reaching this goal, we assumed that crosswalks have a trustworthy pedestrian detection system, which they use to gather information to convey to a nearby Road Side Unit (RSU). This information is then spread along a variety of vehicles of interest in the neighborhood of the source crosswalk using the VANET formed by the vehicles themselves.

To prevent false information spreading, alert messages broadcast by RSUs must be authenticated, and all vehicles (or their On Board Units, OBUs) must validate them. This poses strong security requirements, such as non-repudiation of alert messages, as well as strong real-time requirements, such as minimum message propagation delays among vehicles approaching a crosswalk of interest. Thus, in this crosswalk-vehicle interaction scenario, maximizing efficiency while maintaining safety requirements is mandatory. Considering that RSUs and OBUs must perform several parallel tasks, such as (i) maintaining an Internet connection, (ii) control of car sensors and (iii) driver assistance mechanisms, the authentication procedure can't be a burden.

The Wireless Access in Vehicular Environments (WAVE) is a communication protocol widely used in VANETs. For routing services, WAVE makes use of asymmetric cryptography in order to guarantee both authenticity and non-repudiation ("IEEE Standard for Wireless Access in Vehicular Environments-Security Services for Applications and Management Messages,” 2016). However, asymmetric cryptography, which is 
computationally more intensive than symmetric cryptography, impacts the validation process of messages (Cirne, Zúquete, Sargento, \& Luís, 2019). This is particularly true when used in vehicles' OBUs, given they have limited processing power (Cirne, Zúquete, Sargento, \& Luís, 2019). Consequently, it is associated to delays and to the degradation of applications related with emergency braking signaling (Studer, Bai, Bellur, \& Perrig, 2009).

Considering the crosswalk scenario, we propose a solution mainly based on symmetric ciphers for origin authentication. Our proposal still uses asymmetric ciphers, which are fundamental to guarantee non-repudiation, but minimizes its usage to decrease performance penalties.

\section{RELATED WORK}

Several works, such as (Nambisan, Pulugurtha, Vasudevan, Dangeti, \& Virupaksha, 2009), deal with the detect pedestrians in crosswalks as an improvement to smart cities; yet, often they do not include security measures related to information transmission. Anderson et al. (Anderson et al., 1998) proposed the Guy Fawkes protocol, a message chaining mechanism that includes the hash of the $i+1$-th message in the $i$-th message. However, the loss of a single chain element forces the reestablishment of the full chain. Moreover, the non-repudiation criteria is only accomplished if the first message source can be proved to be the supposed sender (RSU). Perrig et al. (Perrig, Canetti, Tygar, \& Song, n.d.) presented TESLA (Timed Efficient Stream Loss-tolerant Authentication), a protocol for broadcast authentication that relies on both keychains and time synchronization among the different nodes. TESLA uses a combination of easy-to-compute Message Authentication Codes (MACs) and hash functions as an authentication technique, which enables it to take low computation effort to authenticate broadcast streams. By using "time delays", packet buffering and a tight time synchronization, TESLA can tolerate packet losses as well. Perrig et al. also developed TESLA++ (Studer, Bai, Bellur, \& Perrig, 2009) as an improvement of TESLA and a more efficient approach for ad-hoc networks. However, TESLA requires time delays in receivers to properly handle delays, which goes against our real-time requirements.

\section{PROPOSAL}

Taking into account the characteristics of VANETs (mobility, intermittent connectivity, long and variable delay in information delivery), it is important that message validations are performed even if a node loses one or more packages. Our proposal (explained in detail in Section 3.1) makes use of asymmetric ciphers, which are fundamental to guarantee non-repudiation, but minimizes its exploitation to decrease performance penalties.

Our proposal inherits some of the characteristics of the Guy Fawkes Protocol (Anderson et al., 1998)5, therefore we will overview it first. We will as well highlight its limitations regarding fault tolerance to packets losses and vulnerabilities to Man-in-the-Middle (MitM) attacks.

The Guy Fawkes protocol broadcasts two types of packages: Message packages $P_{i}^{m}$ and Validation packages $P_{i}^{v}$ (see Figure 1). The Message package $P_{i+1}^{m}$ contains: (i) a random key $K_{i+1}$ that, when hashed, conforms with Message package $P_{i}^{m}$; (ii) the message contents $M_{i+1}$; and (iii) the hash of the random key $K_{i+2}$ contained in the next Message package, $P_{i+2}^{m}$. The existence of $K_{i+1}$ in $P_{i+1}^{m}$ and its hash in $P_{i}^{m}$ confirms that no message was lost.

A Validation package $P_{i}^{v}$ consists of a hash over the Message package $P_{i}^{m}$ that follows it; it is a commitment used to avoid MitM attacks. Once a message $P_{i}^{m}$ gets accepted and $P_{i+1}^{v}$ gets known by its recipients, only someone knowing both $K_{i+1}$ and $M_{i+1}$ can produce and send $P_{i+1}^{m}$, which creates an authentication chain. The protocol's bootstrap uses digital signatures in order to authenticate the message chain source. 


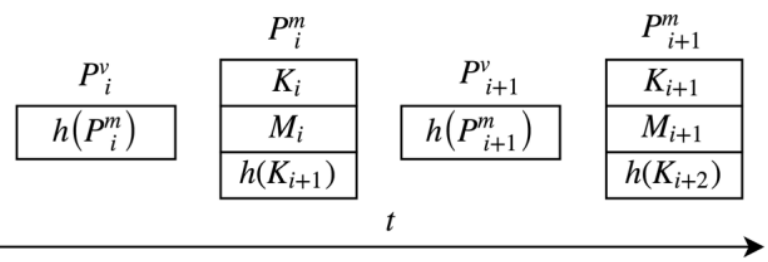

Figure 1. Sequence of packages in the Guy Fawkes protocol. $P_{i}^{v}$ and $P_{i+1}^{v}$ are Validation packages; $P_{i}^{m}$ and $P_{i+1}^{m}$ are Message packages

This protocol presents qualities that can lead to symmetric and efficient cryptography with authenticity and non-repudiation properties. Yet, it does not fit our scenario, since there are several problems with its integration to our use case. Firstly, the high probability of a message being lost in an ad-hoc network. If the recipient misses a packet, one cannot guarantee the authenticity nor the non-repudiation of the next Message packet, because (I) $K_{i}$ and $K_{i+1}$ are independent and (ii) Validation packages are critical to chain validations.

Under some circumstances, it is vulnerable to MitM attacks. In fact, if an attacker is able to deploy a physical barrier to packet broadcasting (e.g. by intercepting the wiring of the antenna of the broadcasting device), it can perform the following attack: (i) block the broadcast of genuine packets from $P_{i}^{m}$ onward; (ii) wait until receiving both $P_{i+1}^{v}$ and $P_{i+1}^{m}$; (iii) Change $M_{i+1}$ in $P_{i+1}^{m}$ to $F_{i+1}$ (fake message) and recompute $P_{i+1}^{v}$ accordingly; (iv) broadcast the tampered packets $P_{i+1}^{v}$ and $P_{i+1}^{m}$ and repeat the process in the following packets. Clock synchronization and fixed reception windows can avoid this problem, but they impose a fixed transmission rate that increases the medium occupancy.

\subsection{Nimble Asymmetric Cryptography (NAC) Protocol}

In general, the IoT sensors used in crosswalks emit a very reduced number of information values from finite sets. Crosswalk sensors, for instance, only transmit one binary message: either someone is using the crosswalk or no one is there. Our proposed solution, called Nimble Asymmetric Cryptography (NAC) and showed in Figure 2, was inspired by the Guy Fawkes protocol but designed in accordance with these characteristics.

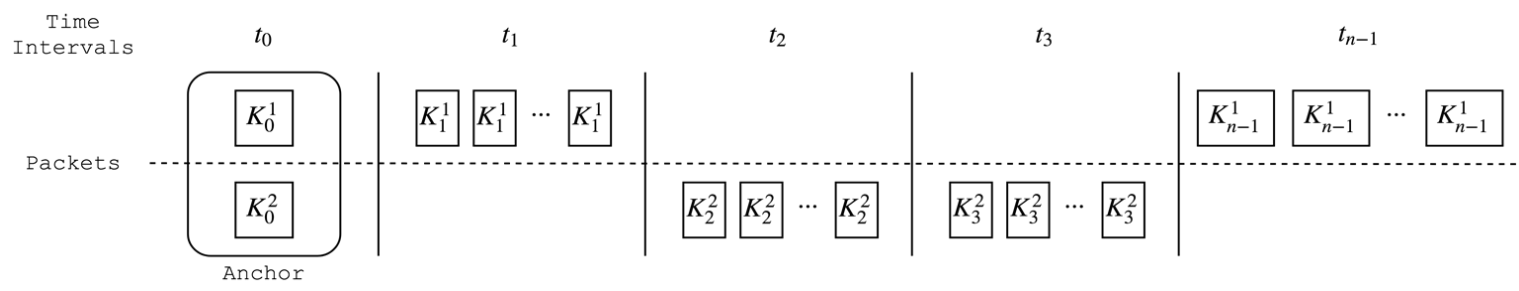

Figure 2. Sequence of messages broadcast with the Nimble Asymmetric Cryptography (NAC). This process is cyclic, at $t_{0}$ the RSU broadcasts an anchor message. At $t_{n}, n$ time intervals after that, the RSU brodacasts a new anchor message, which will contain new $K_{0}^{1}$ and $K_{0}^{2}$ values

NAC uses hash chains of keys. Keys are computed and disclosed in the reverse order of a hashing chain: $K_{i}=h\left(K_{i+1}\right)$. NAC also assumes global time synchronization and that the keys $K_{i}$ are only valid during a particular time frame. The steps of the NAC protocol are:

1. At time interval $t_{0}$ the RSU broadcasts an anchor message (Section 3.2) containing two values, $K_{0}^{1}$ and $K_{0}^{2}$. Both these values are the end of two separate hash chains: $K_{0}^{1}=h^{n}\left(K_{n}^{1}\right)$ and $K_{0}^{2}=h^{n}\left(K_{n}^{2}\right) . K_{n}^{1}$ is a secret key that will be used to advertise the presence of people crossing the crosswalk; similarly, $K_{n}^{2}$ will be used to advertise the opposite.

2. At each time interval $t_{i}$ a new key $K_{i}$ is used in RSU alerts. $K_{i}$ must belong to one of the two hash chains, i.e. it must be either $K_{i}^{1}$ or $K_{i}^{2}$. In order for each OBU to confirm the authenticity of a received $K_{i}$ it must verify if $\mathrm{h}^{i}\left(K_{i}\right)$ equals $K_{0}^{1}$ or $K_{0}^{2}$. This way, each OBU can have implicit guarantees about the existence of people in the crosswalk. Note that, given a previously received (an authentic) message with $K_{i-\delta}$, if $K_{i}$ belongs to the same hash chain it can be checked by computing only $\delta$ hash operations, instead of $i$, because $K_{i-\delta}=h^{\delta}\left(K_{i}\right)$. Observing Figure 2, we can validate $K_{3}$ by checking if $K_{2}=h\left(K_{3}\right)$. 
3. In order to guarantee that OBUs receive at least one message per time interval, the RSU broadcasts the same message more than once during its validity period. The repetition rate can vary according with the relevance of the implicit message for the safety of pedestrians: messages signaling an occupied crosswalk should be repeated more than for a free one.

Note that with NAC it is impossible for an attacker to tamper transmitted messages, contrarily to the Guy Fawkes protocol, because messages are implicitly given by each key hash chain. In other words, the key is the message, $K_{i}$ is also $M_{i}$.

NAC requires clock synchronization between OBUs and RSUs. The OBUs need this synchronization to properly validate $K_{i}$ keys and, therefore, to evaluate the correctness of the implicit message that they convey regarding the current occupancy of the crosswalk. The Global Positioning System (GPS) is not only a navigation system, but also a good option for time-transfer system (Lewandowski, Azoubib, \& Klepczynski, 1999). In our testbed, OBUs and RSUs have GPS, and NAC will use this resource for clock synchronization.

The origin and duration of the time intervals used by NAC do not need to be established globally. In fact, they can be dynamically established by each anchor message.

\subsection{Anchor Messages}

The trust in messages is chained: a recipient trusts in a message because it trusts on some previously received messages. Therefore, there must be some trust anchor, in which a recipient relies, in order to trust the chain that follows it. Such anchor must be a self-protected message with non-repudiation properties and with the two keys, $K_{0}^{1}$ and $K_{0}^{2}$ at the end of the two hash chains. The widely used method to achieve these properties is with digital signatures and public key certificates.

The RSU is subject to two extra tasks: anchor re-sending and anchor updating. New vehicles entering the RSU radio range are not aware of the trust chain. Therefore, the anchor message must be re-sent by the RSU. The length of time interval between anchor disclosures will be subject to further investigation in future work. The RSU is also responsible for updating the anchor messages it sends. When a new vehicle enters the range of an RSU at time $t_{n-1}$, if $\mathrm{n}$ is a sizable number the OBU would take a significant amount of time to compute $h^{n-1}\left(K_{n-1}\right)$ in order to validate $K_{n-1}$. This would create unnecessary overheads. Therefore, it is important that the RSU keeps the size $n$ of key chains under maximum limits, and anchors are updated after $n$ time intervals with new $K_{0}^{1}$ and $K_{0}^{2}$ values, belonging to two new hash chains.

The overhead added by asymmetric cryptography in anchor messages is redeemed by the validation of hash chains, since longer chains reduce the prevalence of overheads due to validations of anchor messages. However, longer hash chains may as well increase the cost of validating hash chains under heavy packet losses; the search for the right balance between chain size and asymmetric usage will be subject of further research.

\subsection{Message Retransmission}

In VANETs, vehicles moving through a city will be constantly switching their access points (RSUs). NAC was designed to address the mobility requirements of the VANET, as we discuss bellow.

1. Anchor Messages: when a recipient (OBU) is in the transmission range of an RSU responsible for a crosswalk, it may already be too late for getting an anchor message directly from the RSU. To minimize this characteristic, NAC uses the spreading of anchor messages between vehicles, maximizing the knowledge of "authenticators" of alert sequences encapsulated within hashing chains.

2. Alert messages: in some cases, due to excessive physical distances or to the presence of physical barriers, OBUs may not receive relevant alert messages direct from the RSU. Consequently, their drivers might not be warned of a possible risk situation. Alert messages can be relayed by OBUs.

NAC covers this possibility with two complementary measures: (i) an RSU broadcasts key $K_{i}$ within time interval $t_{i}$ more than once, for maximizing the nearby reception of alert messages; and (ii) vehicles may relay alert messages, namely the critical ones, up to a maximum range, as they do with anchor messages.

Relatively to alert relaying, vehicles should only relay alerts belonging to the current time frame. Otherwise, the network would be overloaded with bogus messages. Anchor relaying, on the other hand, must occur during the time validity of the anchor, an even a bit before, because new OBUs might be approaching the crosswalk and must be aware of the hash chains to be used. 


\subsection{Using NAC to deal with Other City Sensors}

The NAC protocol conveys implicit messages, being these as many as the number of hashing chains allowed by anchors created by a given source. There are a variety of sensors in a smart city that follow a similar principle, and thus are suitable for being handled using NAC. Traffic lights, for instance, broadcast only three message types: the light is either red, yellow or green. Thus, one can explore NAC with three different hash chains and broadcasting at each time frame the correspondent key for the chain. Other sensors, such carbon dioxide, methane and even trash weight sensors, are often boolean: given a threshold value, they either emit "under the threshold" or "over the threshold" signals. Thus, they could also be handled with NAC.

Concluding, NAC takes advantage of time constraints and the distinctiveness of hash chains in order to achieve broadcast message authentication, while maintaining message loss tolerance and deciphering efficiency. This set of advantages can be of much use in smart cities, where most sensors broadcast a finite set of signals, and in scenarios where message interpretation times can make the difference between life or death.

\section{ACKNOWLEDGMENT}

This work is funded by FCT/MEC through national funds under the project S2MovingCity CMUP-ERI/TIC/0010/2014 and by the European Regional Development Fund (FEDER), through the Competitiveness and Internationalization Operational Programme (COMPETE 2020) of the Portugal 2020 framework, and Financial Support National Public (FCT)(OE); Project, MOBIWISE, POCI-01-0145-FEDER-016426.

\section{REFERENCES}

Anderson, R., Bergadano, F., Crispo, B., Lee, J.-H., Manifavas, C., \& Needham, R. (1998). A New Family of Authentication Protocols. SIGOPS Oper. Syst. Rev., 32(4), 9-20. https://doi.org/10.1145/302350.302353

Cirne, P., Zúquete, A., Sargento, S., \& Luís, M. (2019). The impact of ECDSA in a VANET routing service: Insights from real data traces. Ad Hoc Networks, 90, 101747. https://doi.org/10.1016/j.adhoc.2018.08.017

IEEE Standard for Wireless Access in Vehicular Environments-Security Services for Applications and Management Messages. (2016). IEEE Std 1609.2-2016 (Revision of IEEE Std 1609.2-2013), 1-240. https://doi.org/10.1109/IEEESTD.2016.7426684

Lewandowski, W., Azoubib, J., \& Klepczynski, W. J. (1999). GPS: primary tool for time transfer. Proceedings of the IEEE, 87(1), 163-172. https://doi.org/10.1109/5.736348

Nambisan, S. S., Pulugurtha, S. S., Vasudevan, V., Dangeti, M. R., \& Virupaksha, V. (2009). Effectiveness of Automatic Pedestrian Detection Device and Smart Lighting for Pedestrian Safety. Transportation Research Record, 2140(1), 27-34. https://doi.org/10.3141/2140-03

Perrig, A., Canetti, R., Tygar, J. D., \& Song, D. (n.d.). The TESLA Broadcast Authentication Protocol. 12.

Santos, P. M., Rodrigues, J. G. P., Cruz, S. B., Lourenço, T., d'Orey, P. M., Luis, Y., ... Barros, J. (2018). PortoLivingLab: An IoT-Based Sensing Platform for Smart Cities. IEEE Internet of Things Journal, 5(2), 523-532. https://doi.org/10.1109/JIOT.2018.2791522

Studer, A., Bai, F., Bellur, B., \& Perrig, A. (2009). Flexible, extensible, and efficient VANET authentication. Journal of Communications and Networks, 11(6), 574-588. https://doi.org/10.1109/JCN.2009.6388411 